\title{
COMPOSIÇÃO FLORÍSTICA DE UM TRECHO DE VEGETAÇÃO ARBUSTIVA CADUCIFÓLIA, NO MUNICÍPIO DE IBIMIRIM, PE, BRASIL
}

\author{
Maria Jesus Nogueira Rodal' \\ Ladivania Medeiros do Nascimento' \\ André Laurênio de Melo'
}

Recebido em 01/07/1998. Aceito em 17/12/1998

\begin{abstract}
RESUMO - (Composição florística de um trecho de vegetação arbustiva caducifólia, no município de Ibimirim. PE. Brasil). Com o objetivo de contribuir para o conhecimento da estrutura taxonômica e identidade florística da vegetação arbustiva caducifólia das chapadas sedimentares do semi-árido de Pernambuco, foi realizado o levantamento da flora angiospérmica de uma área situada no município de Ibimirim. PE. Em 343 coletas, foram identificados 139 táxons. distrihuídos em 92 gêneros e 38 famílias. As famílias com maior riqueza foram: Euphorbiaceae, Fabaceae, Caesalpiniaceae e Mimosaceae com 42\% e 33\% das espécies e gêneros. respectivamente. A partir da análise de 831 espécies listadas em 15 levantamentos florísticos, distribuídos em 19 áreas (Ceará. Pemambuco. Piauí e Sergipe), identificou-se que o maior número de espécies em comum ocorreu com levantamentos localizados em chapadas sedimentares e caatingas instaladas no cristalino, e menor, com o levantamento de cerrado no Estado do Piaú. A partir dos aspectos florísticos. funcionais e grau de deficiência hídrica da área de estudo, a vegetação foi considerada como pertencente à caatinga.
\end{abstract}

Palavras-chave - caatinga, flora angiospérmica, região semi-árida

\begin{abstract}
Floristic composition of the shrubland decidual vegetation at Ibimirim district, Pernambuco, Brazil). A survey of the flora was carried out on deciduous shrubby vegetation from the sedimentary plateaus of Ibimirim. Pernambuco State. Brazil. In 343 collections, 139 taxa were identified, these being distributed in 92 genera and 38 families. The families richest in species were: Euphorbiaceae. Fabaceae. Caesalpiniaceae and Mimosaceae, with $42 \%$ and $33 \%$ of the species and genera, respectively. Data analysis of 831 species listed in 15 floristic surveys from 19 sites (Ceará. Pernambuco, Piauí and Sergipe States) showed that the area studied has the most species in common with sites located on sedimentary plateaus and caatingas that overlie cristaline rock. and the fewest in common with the cerrado vegetation of Piaui State. The vegetation is classified as caatinga based on the flora. functional aspects and degree of water deficiency.
\end{abstract}

Key words - caatinga, Angiosperm flora, semi-arid region

\section{Introdução}

Segundo Andrade-Lima (1981) e Mello Netto et al. (1992), a vegetação de caatinga recobre conjunto de diferentes paisagens do semiárido nordestino, onde, na quase totalidade das espécies, predomina a caducifolia sobre as demais formas de resistência à seca. Streillein (1982) observou que na caatinga ocorrem desde associações vegetacionais simples a extremamente complexas, em função da composição florística, abundância das espécies, altura e espécies

\footnotetext{
'Departamento de Biologia, UFRPE, Rua Dom Manoel de Medeiros s/n. CEP 52171-900, Recife, PE. Brasil
} 
características, salientando ainda que inúmeros tipos vegetacionais são freqüentemente encontrados em áreas bastante pequenas, criando verdadeiro mosaico vegetacional. Para Fernandes (1996), a caatinga e o carrasco são os tipos vegetacionais característicos do semi-árido, enquanto as manchas de floresta úmida ou seca, e as variações de cerrado seriam vegetações residuais de períodos climáticos mais úmidos.

Técnicos do RADAMBRASIL comentaram que a duração e a intensidade do período seco e o excedente hídrico são fundamentais na compreensão das diferentes fitofísionomias da caatinga, porém todas elas se caracterizariam por apresentar maior proporção de microfanerófitos e caméfitos com adaptações ao período desfavorável, além de plantas herbáceas com brotos foliares protegidos pela folhagem morta (hemicriptófitas), pelo solo (geófitas) e ervas anuais (terófitas), como resultado de seleção de formas de vida adaptadas a esses ambientes. Tais aspectos revelariam, como observou Reis (1976), a influência fundamental do clima na vegetação de caatinga.

Andrade-Lima (1981) considerou que a caatinga está circunscrita, aproximadamente, no espaço situado a partir da isoeta de $1.000 \mathrm{~mm} / \mathrm{ano}$. Nos últimos anos, autores como Fernandes \& Bezerra (1990) passaram a adotar a isoeta de $800 \mathrm{~mm} / \mathrm{ano}$ como limite para a caatinga. Recentemente, técnicos da FUNCEME redimensionaram o semi-árido e, ao invés de adotar um único parâmetro - a isoeta de $800 \mathrm{~mm} / \mathrm{ano}$, consideraram um conjunto de fatores, como relevo, solo, litologia e hidrologia de superfície e subsuperfície, para circunscrevê-lo (Souza et al. 1994). Concluíram que o semi-árido ocupa sete geoambientes, no total de $788.064 \mathrm{~km}^{2}$, em áreas cuja precipitação média anual varia entre 400 a $800 \mathrm{~mm}$ e onde, além da caatinga, ocorrem alguns tipos vegetacionais transicionais com outras formações ocorrentes na região Nordeste. Reis (1976) e Nimer (1979) consideraram que a irregularidade espacial e temporal da distribuição das chuvas, associada às baixas precipitações, temperaturas elevadas e fortes taxas de evaporação e evapotranspiração, são as principais características do semi-árido nordestino
Do ponto de vista geológico-estrutural, o semi-árido apresenta significativa parcela de terrenos da depressão sertaneja. Trata-se do setor nordeste do escudo cristalino brasileiro, de origem pré-cambriana, constituído principalmente por rochas metamorfizadas, não raro impregnadas por rochas eruptivas graníticas e granodioríticas, estando em outras partes constituído por gnaisses (Andrade 1977; Moreira 1977).

Outra unidade geossistêmica de destaque, por sua extensão, é a dos planaltos sedimentares. Sua maior área de ocorrência está na bacia sedimentar do meio norte, além da bacia do Araripe (Ceará/ Pernambuco), Tucano-Jatobá (Bahia/Pernambuco), e de outras de pequenas dimensões no Ceará, Rio Grande do Norte e Paraíba (Souza et al. 1994). Tratam-se de bacias paleozóicas ou mesozóicas que incluem chapadas, chapadões, cuestas, baixos platôs, comumente capeadas por arenito, onde predominam Latossolos e Areias Quartzosas Distróficas. Os índices pluviométricos são mais elevados no planalto do Piauí e chapada do Araripe (600 a 900mm), e na bacia do Jatobá decrescem abaixo de $600 \mathrm{~mm}$ (Souza et al. 1994).

Apesar de amplamente conhecida a correlação entre a distribuição da caatinga e a depressão sertaneja (Rizzini 1979), autores como Andrade-Lima (1981) observaram que esta deve ser aceita, mas com algumas ressalvas, em vista da ocorrência de caatinga na chapada do Apodi (Andrade-Lima 1964; Figueiredo 1987) e no planalto da Borborema (Alcoforado-Filho 1993). Enquanto a flora e a vegetação da caatinga situada sobre a depressão sertaneja são relativamente conhecidas (Tavares et al. 1969a, 1969b; 1970; 1974a, 1974b; 1975; Drumond et al. 1979; Gomes 1979; Albuquerque et al. 1982; Lyra 1982; Souza 1983; Santos 1987; Ferreira 1988; Oliveira et al. 1988; Fonseca 1991; Rodal 1992; Alcoforado-Filho 1993; Araújo et al. 1995; Ferraz et al. 1998), aquelas localizadas em bacias sedimentares, em áreas do semi-árido, só ultimamente vêm despertando maior interesse (Rodal 1983; Oliveira et al. 1997; Araújo et al. 1998; Araújo et al. 1998; Rodal et al. 1998; Figueirêdo et al. no prelo). 
A classificação da vegetação arbustiva caducifólia das chapadas sedimentares, especialmente no Piauí, Ceará, Pernambuco e Bahia, tem gerado controvérsias. No caso da vegetação arbustiva caducifólia que recobre o topo do planalto do Ibiapaba, Ceará, Araújo et al. (1998) observaram que a vegetação de carrasco que ali ocorre é distinta do ponto de vista florístico, da caatinga instalada sobre o cristalino. No caso do Piauí e Pernambuco, autores como VasconcelosSobrinho (1941), Egler (1951), Andrade-Lima (1960, 1978) e Emperaire (1991) consideraram que a vegetação caducifólia que recobre as chapadas é um tipo de caatinga, apesar da flora particular, bastante ligada a solos de origem sedimentar altamente arenosos. Para esses autores, a identidade com a caatinga seria confirmada pela presença de comunidades vegetais, comandadas por ambiente climático geral da região semi-árida. Por outro lado, Oliveira et al. (1997) e Figueirêdo et al. (no prelo) reconhecem a individualidade daquela flora.

Assim, com o objetivo de contribuir para o conhecimento da flora angiospérmica das chapadas sedimentares, foi realizado o levantamento florístico de um trecho de vegetação arbustiva caducifólia, localizada no município de Ibimirim. Pernambuco, identificando sua estrutura florística e realizando comparações florísticas com outras formações vegetacionais nordestinas, especialmente aquelas localizadas no semi-árido.

\section{Material e métodos}

A área foi selecionada por representar a flora e vegetação típicas das chapadas sedimentares de Pernambuco localizadas no semi-árido. Situa-se no municipío de Ibimirim em área de dez hectares, entre as coordenadas $8^{\circ} 39^{\prime}$ a $8^{\circ} 41^{\prime} \mathrm{S}$ e $37^{\circ} 35^{\prime}$ a $37^{\circ} 37^{\prime} \mathrm{W}$, em altitude de $600 \mathrm{~m}$. De acordo com Andrade-Lima (1966), a vegetação pode ser classificada como arbustiva. A sinúsia arbórea é descontínua, a arbustiva é densa, exceto nas áreas de afloramentos rochosos, com tapete herbáceo praticamente inexistente, exceto nas áreas mais abertas.

Tomando-se como base os dados de temperatura média compensada e precipitação da estação meteorológica da sede do município de Floresta (Banco de dados climatológicos do Departamento de Recursos Minerais, SUDENE) situada a $30 \mathrm{~km}$ de distância da área de estudo, elaborou-se o diagrama pluviotérmico (Walter \& Leith 1964). Observam-se praticamente oito meses com déficit hídrico, com temperatura média anual variando de $25^{\circ} \mathrm{C}$ a $30,9^{\circ} \mathrm{C}$ (Fig. 1).

A área localiza-se na bacia sedimentar do Jatobá. uma das mais completas seqüências estratigráficas que afloram no país, em terrenos da formação Marizal, datada do Cretáceo Inferior (Brito 1979). Tratam-se de camadas basais de sequiência sedimentar, repousando discordantemente sobre o cristalino, formando arenitos, folhelhos, siltitos e conglomerados. Os solos são arenosos e profundos, do tipo Areia Quartzosa Distrófica (Jacomine et al. 1973; SUDENE 1979; Dantas 1980), com drenagem incipiente, o que, segundo Ferreira \& Cadier (1978), se deve ao baixo escoamento superficial, ligado à permeabilidade e profundidade do solo, tipo de rocha e relevo.

As coletas mensais aleatórias de material botânico da flora angiospérmica foram realizadas entre abril de 1994 e dezembro de 1996, seguindo as técnicas usuais para coleta de material botânico (Bridson \& Forman 1992). A identificação foi realizada através de morfologia comparada com auxílio de lupa estereoscópica, chaves de identificação e bibliografia especializada (Martius \& Eichler 1840/1906; Barroso et al. 1978, 1984, 1986), analisando exsicatas depositadas nos herbários do Departamento de Biologia da Universidade Federal Rural de Pernambuco (PEUFR), onde as exsicatas foram tombadas, e da Empresa Pernambucana de Pesquisa Agropecuária (Dárdano de Andrade-Lima/IPA). A confirmação e/ou complementação das identificações foi realizada por especialistas do Brasil e do exterior. As espécies foram organizadas por famílias, segundo o sistema de Cronquist (1988), incluindo-se informação sobre o hábito (Font-Quer 1977). A grafia dos gêneros e a autoria das espécies foram verificadas através de Stafleu \& Cowan (1976/1988), Brummitt (1992) e Brummitt \& Powell (1992). 


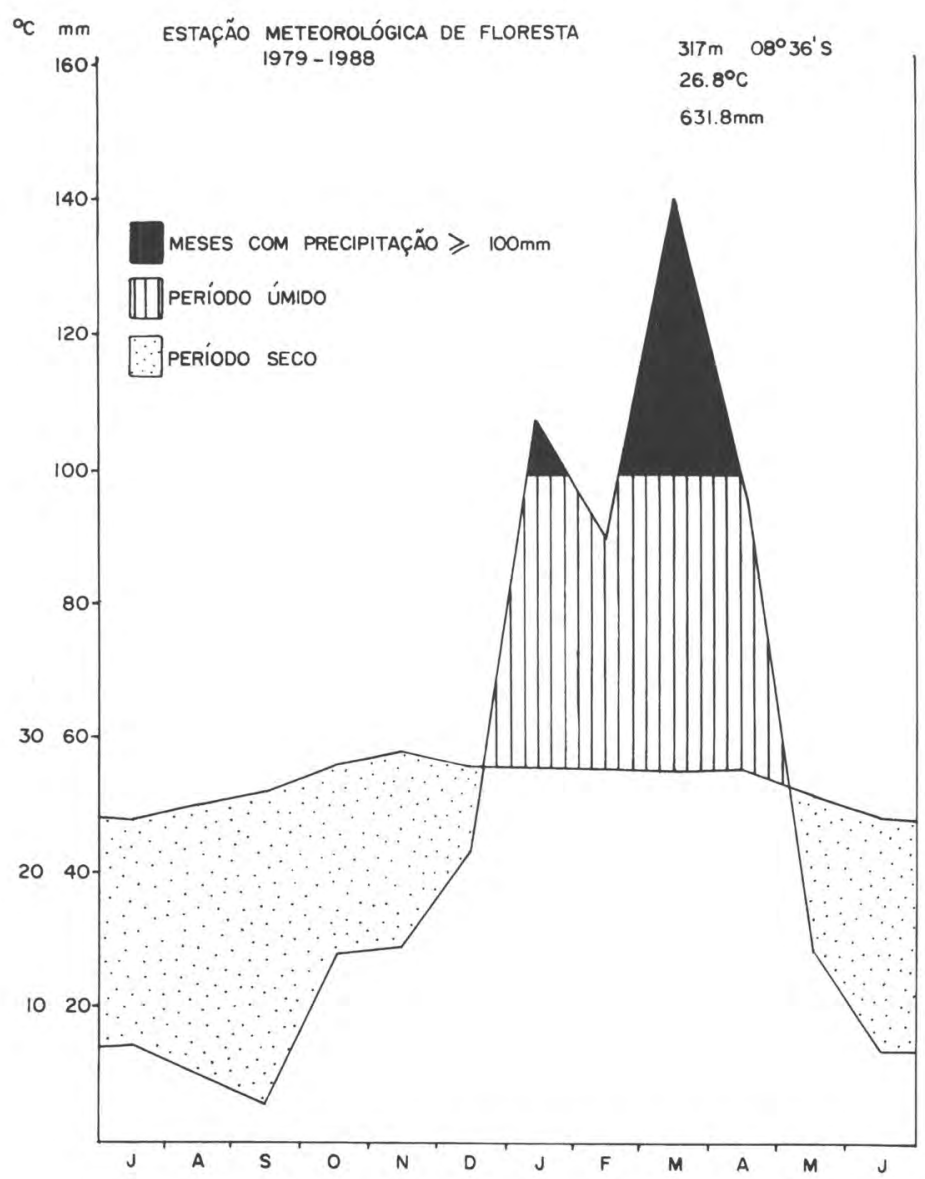

Figura 1. Diagrama pluviotérmico do município de Floresta, Pernambuco

Os resultados da lista florística foram comparados a nove levantamentos realizados em diferentes tipos fitosionômicos de caatinga, instalados em áreas do cristalino (Lyra 1982; Souza 1983; Fotius \& Sá 1985; Santos 1987; Rodal 1992; Alcoforado-Filho 1993; Araújo et al. 1995; Medeiros 1995; Ferraz et al. 1998), quatro em áreas de vegetação arbustiva caducifólia e perenifólia sobre chapadas sedimentares (Rodal 1992; Andrade 1996; Oliveira et al. 1997; Figueirêdo et al. no prelo), um em carrasco (Araújo et al. 1998) e um em cerrado (Castro 1994). A Fig. 2 mostra a localização geográfica da área de estudo e das demais áreas comparadas.

\section{Resultados e discussão}

A Tab. 1 lista 139 táxons distribuídos em 92 gêneros e 39 famílias, dos quais $89 \%$ identificados no nível de espécie e $11 \%$ no nível de gênero. Desse total, $18,7 \%$ são árvores, $48,2 \%$ arbustos e subarbustos, $12,2 \%$ ervas, $16,5 \%$ trepadeiras e/ou lianas e $4,4 \%$ epífitas.

Destacam-se as famílias Euphorbiaceae com 22 espécies, seguida por Caesalpiniaceae (14) e Mimosaceae (13). A elevada riqueza dessas famílias no semi-árido pode também ser observada na Tab. 2, confirmando as observações de Rodal (1992). Gentry (1995), por sua vez, também indica 
a importância da família Euphorbiaceae em florestas decíduas neotropicais.

Como elementos arbóreos que mais se destacaram na paisagem, pelo maior porte, podese citar: Spondias tuberosa (Anacardiaceae), Commiphora leptophloeos (Burseraceae), Pilosocereus pachycladus var. pernambucensis (Cactaceae), Caesalpinia microphylla (Caesal- piniaceae), Cnidosculus obtusifolius (Euphorbiaceae) e Piptadenia obliqua (Mimosaceae). Entre as populações mais abundantes do estrato arbustivo ocorrem: Senna rizzinii (Caesalpiniaceae), Croton micans e Jatropha mutabilis (Euphorbiaceae), Bocoa mollis (Fabaceae) e Pavonia glazioviana (Malvaceae). No estrato herbáceo, destaque para as populações abundantes de: Ipomoea verbascoidea (Convolvulaceae), Sida

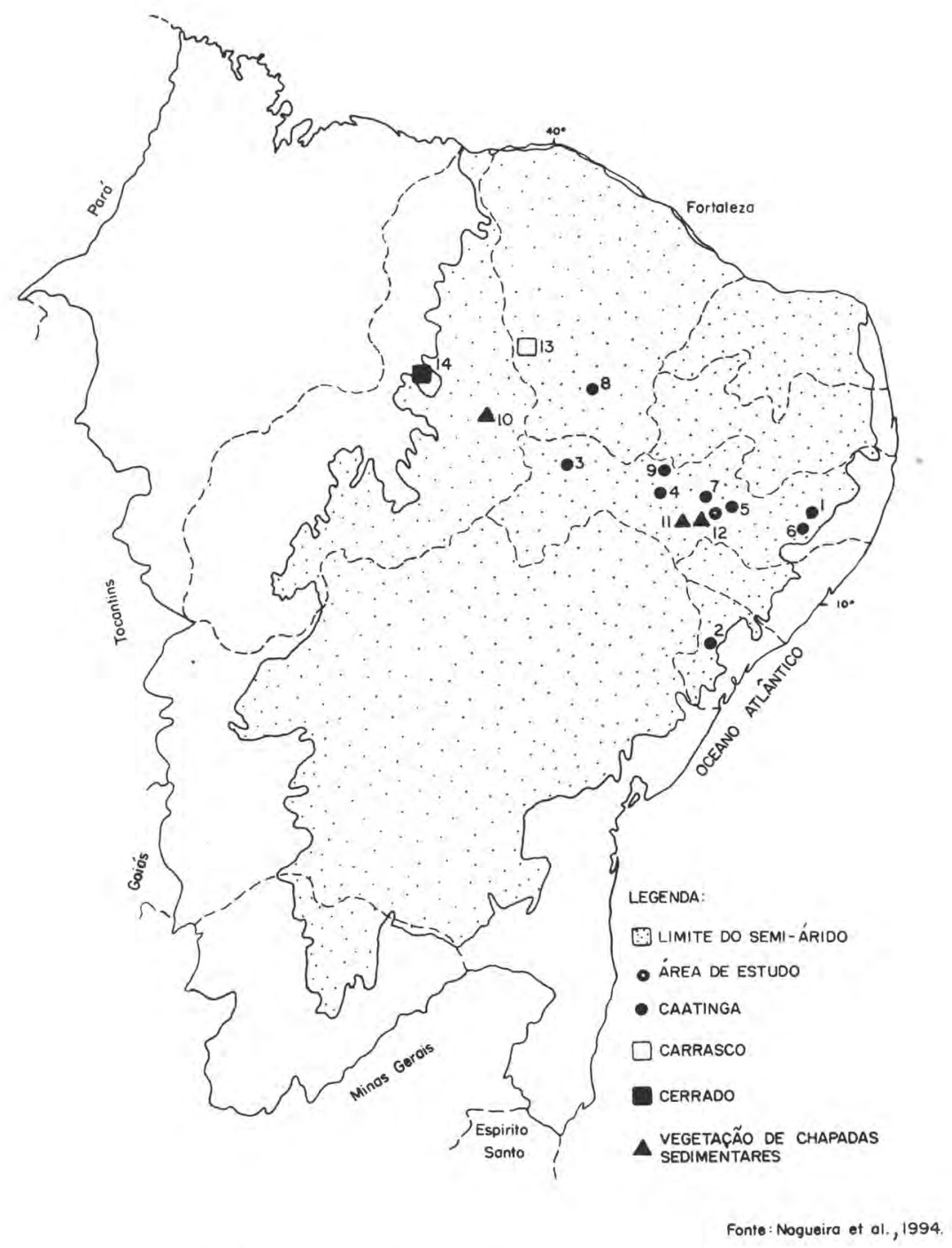

Figura 2. Localização geográfica da área de estudo de outros levantamentos no nordeste do Brasil. Caatinga (1. Lyra 1982;2. Souza 1983; 3. Fotius \& Sá 1985; 4. Santos 1987; 5. Rodal 1992; 6. Alcoforado-Filho 1993; 7. Araújo et al. 1995; 8. Medeiros 1995 e 9. Ferraz et al. 1998); Vegetação de chapadas sedimentares (10. Oliveira et al. 1997; 11. Andrade 1996; 12. Figueiredo et al. prelo); Carrasco (13. Araújo et al. 1998); Cerrado (14. Castro 1994). 
Tahela 1. Lista das famílias e espécies, com especialista e sigla de herhário. encontradas na vegetação de caatinga arbustiva no município de Ibimirim. Pernambuco com seu respectivo coletor, número de coletal ( $\mathrm{N}^{\circ}$ ), hábito (HAB) e sua ocorrência em outros levantamentos. CRIS - vegetação de caatinga sobre o cristalino (Lyra 1982; Souza 1983; Fotius \& Sá 1985; Santos 1987; Rodal 1992; Alcoforado-Filho 1993; Araújo et al. 1995; Medeiros 1995; Ferraz et al. 1998): CAA - vegetação arbustiva caducifólia e ou perenifólia sobre sedimento (Rodal 1992; Andrade 1996; Oliveira et al. 1997; Figueirêdo et al. prelo); CAR - carrasco (Araújo et al. 1998); CER - cerrado (Castro 1994); ARV - árvore; ARB - arbusto; SUB - subarbusto: TR - trepadeira; LIA - liana; ER - erva e EP - epífita. * espécies listadas apenas na área de estudo. Os números indicam a quantidade de levantamentos em que as espécies estão citadas.

\begin{tabular}{llllllll}
\hline Família/Espécie/Identificador/Herbário & Coletor & $N^{\circ}$ & HAB & CRIS & CAA & CAR & CER \\
\hline
\end{tabular}

1. Amaranthaceae

1. Froelichia humboldtiana Roem \& Schultz*

2. Gomphrena vaga Mart**

$\begin{array}{lrr}\text { Menezes } & 47 & \text { ER } \\ \text { Rodal } & 626 & \text { ER }\end{array}$

2. Anacardiaceae

3. Schinopsis brasiliensis Engl.

3. Annonaceae

4. Rollinia leptopetala

(R.E. Fries) Safford

$\begin{array}{llllll}\text { Tschá } & 56.3 & \text { ARV } & 1 & & \\ \text { Laurênio } & 295 & \text { ARV } & 1 & 3 & 1\end{array}$

4. Apocynaceae

5. Allamanda blanchetii A. DC.

6. Aspidosperma prrifolium Mart.

Marcon

159

M.C. Tschá

5. Asteraceae (J.A. Bastos-PEUFR; N. Hind-K)

7. Conocliniopsis prasiifolia (DC.)

R.M. King \& H. Rob.

8. Tridax procumbens L. *

9. Vernonia chalybaea Mart. ex DC.

10. V. scorpiodis (Lam.) Pers. *

6. Bignoniaceae (C. Sothers-K;

L. G. Lohman-MO)

11. Anemopaegma laeve DC.

12. Arrabidaea corallina (Jacq.) Sandwith

13. A. cf. limae A.H.Gentry

14. Clytostoma binatum (Thunb.) Sandwith*

7. Boraginaceae (J. Kallundi-NY;

N. Taroda-SJRP)

15. Cordia globosa (Jacq.)

Humb.. Bonpl. \& Kunth

16. Cordia leucocephala Moric.

17. C. rufescens A.DC.*

18. Cordia sp.

19. Heliotropium indicum L.*

20. H. procumbens Mell.*

\section{Bromeliaceae}

21. Neoglaziovia variegata

(Arruda) Mez

22. Tillandsia recurvata $\mathrm{L}$.

23. T. usneoides (L.) L.*

\section{Cactaceae}

24. Cereus janacaru DC.

25. Opuntia inamoena K. Schum.

26. Pilosocereus pachycladus

var. pernambucensis (F. Ritter) Zappi

$\begin{array}{lrrr}\text { Silva } & 102 & \text { ER } & 1 \\ \text { Sales } & 628 & \text { ER } & \\ \text { Gomes } & 41 & \text { SUB } & 1 \\ \text { Gomes } & 70 & \text { SUB } & \end{array}$

$\begin{array}{lrrrr}\text { Laurênio } & 188 & \text { TR } & 1 & \\ \text { Silva } & 151 & \text { TR } & 2 & \\ \text { Gomes } & 221 & \text { TR } & & 1 \\ \text { Gomes } & 70 & \text { TR } & & \end{array}$

$\begin{array}{lllll}\text { Tschá } & 562 & \text { ARB } & 6 & 1\end{array}$

Laurênio

ARB

4

3

Oliveira

Laurênio

142

ARB

SUB

$599 \quad$ ER

Laurênio

266

EP

2

2

Pimentel

96

EP

Pimentel

95

EP

Pimentel

Pimentel

Figueirêdo

9

ARV

ER

102

$\mathrm{ARB}$ 
Tahela I. (continuação)

Família/Espécie/Identificador/Herbário

Coletor

$\mathrm{HAB}$ CRIS

CAA CAR CER

10. Caesalpiniaceae (J.E. Gomes-PEUFR: R. Barneby-NY)

27. Bauhinia acuruana Moric.

28. Caesalpinia ferrea Mart. \& Tul.

29. C. microphylla Mart. ex G. Don.

30. C. prramidalis Tul.

31. Chantecrista nictitans (L.) Moench*

32. C. repens var. multiflora (Benth.) H.S. Irwin \& Barneby*

33. Poeppigia procera C. Presl

34. Senna acturuensis (Benth.)

H.S. Irwin \& Barneby var. acuruensis*

35. S. macranthera (Vogel) H.S. Irwin \& Barneby*

36. S. accidentalis (L.) Link*

37. S. ri-imii H.S. Irwin \& Barneby

38. S. spectabilis (DC.)

H.S. Irwin \& Barneby var, excelsa (Schrad.) H.S. Irwin \& Barneby

39. S. trachypus (Benth.) H.S. Irwing \& Barneby

11. Capparaceae (M.B. Costa e Silva -PEUFR)

40. Capparis flexuosa (L.) L.

12. Convolvulaceae

41. Evolvulus sericeus Swartz*

42. Ipomoea verbascoidea Choisy*

43. Jacquemontia of. bahiensis O'Donell*:

44. Merremia sp.

13. Cucurbitaceae

45. Cavaponia sp.

14. Erythroxylaceae

46. Ervthmx/um revolutum Mart.

15. Euphorbiaceae (A. Laurênio, M.F.A. Lucena e Santos - PEUFR)

47. Acalypha multicaulis Müll.Arg.

48. Chameresyce thymifolia (L.) Millsp.

49. Cnidoscolus ef. bahianus (Ule) Pax \& K. Schum.

50. C. obursifolius Pohl

51. C. curercifolius Pohl ex Baill.

52. C. urens (L.) Arthur

53. Croton micans (Swartz \& Mind.) Miill. Arg.

54. C. conduplicatus Kunth

55. C. sonderianus Müll. Arg.

56. C. rhaminifolius (Baill.) Muill. Arg. *

57. C. grewioides Baill**

58. Croton sp. 1

59. Croron sp. 2

60. Croton sp. 3

\begin{tabular}{|c|c|c|c|}
\hline Tschá & 267 & ARB & \\
\hline Laurênio & 315 & ARV & 6 \\
\hline Laurênio & 87 & ARV & 2 \\
\hline Laurênio & 12 & ARV & 8 \\
\hline Menezes & 49 & SUB & \\
\hline Rodal & 601 & SUB & \\
\hline Laurênio & 78 & ARV & \\
\hline Gomes & 37 & ARB & \\
\hline Rodal & 603 & ARB & \\
\hline Rodrigues & 15 & ARB & \\
\hline Rodal & 577 & ARV & 1 \\
\hline Silva & 148 & ARB & \\
\hline Rodal & 599 & ARB & \\
\hline Pimentel & 13 & ARV & 7 \\
\hline Figueirêdo & 14 & $\mathrm{TR}$ & \\
\hline Rodal & 628 & TR & \\
\hline Andrade & 102 & TR & \\
\hline Andrade & 10.3 & $\mathrm{TR}$ & \\
\hline Laurênio & 292 & TR & \\
\hline Laurênio & 293 & $A R B$ & \\
\hline Rodal & 611 & ARB & 4 \\
\hline Sales & 629 & ER & \\
\hline Rodal & 712 & ARB & 3 \\
\hline Rodal & 713 & ARV & \\
\hline Laurênio & 31.3 & ARV & 1 \\
\hline Rodal & 714 & $\mathrm{ARB}$ & 3 \\
\hline Rodal & 731 & $\mathrm{ARB}$ & 1 \\
\hline Inácio & 10 & ARB & 1 \\
\hline Inácio & 175 & ARB & 5 \\
\hline Rodrigues & 12 & ARB & \\
\hline Rodal & 730 & ARB & \\
\hline Pimentel & 93 & ARB & \\
\hline Laurênio & 288 & ARB & \\
\hline Laurênio & 309 & $A R B$ & \\
\hline
\end{tabular}


Tahela 1. (continuação)

Família/Espécie/Identificador/Herbário

Coletor

$\mathrm{N}^{\mathrm{o}}$

$\mathrm{HAB}$

CRIS

CAA

CAR CER

15. Euphorbiaceae (A. Laurênio, M.F.A. Lucena e Santos - PEUFR)

61. Dalechampia sp.

62. Euphorbia comosa Vell.

63. Jatropha molissima (Pohl) Baill.

64. J. mutabilis (Pohl) Baill.

65. J. ribifolia (Pohl) Baill.

66. Manihot dichotoma Ule

67. Sebastiania brasiliensis Spreng.

68. Stillingia trapezoidea Ule*

Sales

Laurênio

Pimentel

Laurênio

Lucena

Sales

Rodal

Laurênio

16. Fabaceae (G.Lewis-K; R.Barneby-NY)

69. Bocoa mollis (Benth.) Cowan

70. Centrosema virginianum (L.) Benth.

71. Chaetocalyx scandens (L.) Urb. var. pubescens (DC.) Rudd*

72. Cratylia mollis Mart. ex Benth.

73. Crotalaria holosericea Nees ex Mart.*

74. C. hahiensis Windler \& Skinner.

75. Dalbergia catingicola Harms*

76. D. cearensis Ducke

77. Dioclea grandiflora Mart. ex Benth.

78. Indigofera suffruticosa Mill.*

79. Luetzelburgia sp.

80. Zormia diphylla (L.) Pers.

17. Lamiaceae (R.M. Harley-K)

81. Eriope sp

82. Hyptis martiusii Benth.

18. Loganiaceae

83. Struchuos rubiginosa DC.

19. Loranthaceae (B. Stannard-K)

84. Orvcthanthus sp.

85. Struthanthus polyrhizus Mart.*

20. Malpighiaceae (C.Sothers-K;

W.R.Anderson-MICH)

86. Banisteriopsis muricata (Cav.) Cuatr.*

87. Byrsonima gardneriana A. Juss.

88. Heteropterys trichanthera A.Juss.*

89. Stigmaply.llom auriculatum (Cav.) A. Juss.:

90. S. paralias A. Juss.

21. Malvaceae (G.S. Baracho-UFP: P.A. Fryxell-TEX)

91. Herissantia crispa (L.) Briz.*

92. H. tiubae (K.Schum.) Briz.

93. Paronia glazioviana Gürke

94. P. varians Moric.*

95. Sida cordifolia L.

96. S. galheirensis Ulbr.

22. Mimosaceae (G. Lewis-K; R. Barneby-NY)

97. Acacia sp.

98. Chloroleucon foliolosum (Benth.) G.P. Lewis
Inácio

Laurênio

Laurênio

Laurênio

Laurênio

Laurênio

Gomes

Tschá

Tschá

Lucena

Inácio

Laurênio

Laurênio

Silva

Tschá

Sales

Sales

Laurênio

Sales

Gomes

Tschá

Gomes

Silva

Silva

Laurênio

Pimentel

Andrade

Rodrigues

Gomes

Laurênio

531

69

10

287

166

566

641

307

TR

SUB

ARV

ARB

ARB

ARV

ARV

ARB

108

84

66

ARB

TR

TR

88

$A R B$

ARB

SUB

ARV

ARV

LIA

ARB

107 ARB

114 ARV

280

SUB

10.3 SUB

399

TR

569 EP

632 EP

68

TR

ARB

TR

121 TR

222 TR

14 ER

152 ARB

ARB

104 SUB

17

ARB

SUB

2

1

I

12

227

ARV

ARB
1

2

21

$\begin{array}{lll}1 & 1 & \\ 8 & 3 & \\ 2 & 3 & \\ 4 & 1 & \\ 1 & 1 & 1\end{array}$

1

2

l 1

21

1

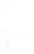


Tahela I. (continuação)

Família/Espécie/Identificador/Herbário

Coletor

$\mathrm{N}^{\circ} \quad \mathrm{HAB}$

CRIS

CAA

CAR CER

22. Mimosaceae (G, Lewis-K;

R. Barneby-NY)

99. Mimosa adenophylla Taub.

var. mitis Barneby*

100. M. aremosa (Willd.) Poir.

101. M. genmmulata Barneby*

102. M. tenuiflora (Willd.) Poir.

103. M. guaranitica Chad. \& Hesse**

104. Mimosa sp. I

105. Mimosa sp. 2

106. Parapiptadenia zehumeri (Harms) M.P. Lima \& H.C. Lima

107. Piptadenia obliqua (Pers.) J.F. Macbr.

108. P. stipulacea (Benth.) Ducke

109. Piptadenia sp.

$\begin{array}{lclll}\text { Sales } & 567 & \text { ARB } & & \\ \text { Rodal } & 648 & \text { ARB } & \text { I } & \\ \text { Gomes } & 157 & \text { ARB } & & \\ \text { Gomes } & 153 & \text { ARV } & 3 & 1 \\ \text { Rodal } & 629 & \text { ARB } & & \\ \text { Gomes } & 156 & \text { ARB } & & \\ \text { Freire } & 96 & \text { ARB } & 3 & 1 \\ \text { Gomes } & 155 & \text { ARV } & 3 & 3 \\ \text { Rodal } & 493 & \text { ARV } & & 3 \\ \text { Tschá } & 265 & \text { ARB } & 4 & 2 \\ \text { Tschá } & 397 & \text { ARB } & & \\ \text { Rodal } & 627 & \text { ER } & 1 & \\ & & & & \\ \text { Laurênio } & 282 & \text { ARB } & & \\ \text { Sales } & 6.35 & \text { ARB } & & \\ \text { Pimentel } & 87 & \text { ARB } & & \\ \text { Tschá } & 564 & \text { ARV } & & \\ \text { Gomes } & 223 & \text { TR } & & \end{array}$

23. Molluginaceae

110. Mollugo verticillata $\mathrm{L}$.

24. Myrtaceae (G.M. Barroso-RB)

111. Campomanesia aromatica (Aubl.) Griseb.

112. Eugenia punicifolia (Humb., Bonpl. \& Kunth) DC.

113. Psidium myrsinoides Berg.*

25. Nyctaginaceae

114. Guapira laxa (Netto) Furlan

26. Passifloraceae

115. Passiflora luetzelburgii Harms

27. Portulacaceae

116. Portulaca aff. elatior Mart."

117. P. cf. grandiflora Hook.*

28. Rhamnaceae

118. Ziziphus joazeiro Mart.

29. Rubiaceae (E.B. Souza-PEUFR)

119. Mirracarpus hirtus (L.) DC.*

120. Richardia brasiliensis Gomes*

121. R. grandiflora (Cham.

$\&$ Schlecht.) Steud

30. Rutaceae (J.Kallunki-NY)

122. Balfouradendron molle (Miquel) Pirani

Villarouco

22

ER

Sales $\quad 563$ ER

Rodal $689 \quad$ ARV

Sales

Tschá

Gomes

671

ER

47

Rodal

ARV

Sales

123. Cardiospermum anomalum Cambess."

124. C. corindum L.

125. Serjania glabrata Kunth

126. Serjania lethalis A.St.-Hil

Oliveira

Oliveira

$278 \quad$ TR

Oliveira

11

141

TR

TR

Silva

153 ARV

4

127. Sidemilon obtusifolium

(Roem. \& Schult.) T.D. Penn.

33. Solanaceae (S. Knapp-K)

128. Solanum haturitense Huber

129. Solamum flaccidum Vell.*

Tschá

I


Tahela 1. (continuação)

\begin{tabular}{|c|c|c|c|c|c|c|c|}
\hline Família/Espécie/Identificador/Herbário & Coletor & $\mathrm{N}^{\circ}$ & $\mathrm{HAB}$ & CRIS & CAA & CAR & CER \\
\hline \multicolumn{8}{|l|}{ 34. Sterculiaceae } \\
\hline 1.31. Melochia tomentosa $\mathrm{L}$. & Lira & 22 & SUB & 4 & & & \\
\hline 132. Waltheria ferruginea A St.-Hil. & Pimentel & 105 & SUB & & 3 & 1 & \\
\hline 13.3. W. anericana $\mathrm{L}$. & Gomes & 71 & SUB & & 1 & & \\
\hline \multicolumn{8}{|l|}{ 35. Tiliaceae } \\
\hline 134. Prockia crucis P.Browne ex L. & Laurênio & 310 & ARB & 1 & & & \\
\hline \multicolumn{8}{|l|}{ 36. Turneraceae } \\
\hline 135. Piriqueta sp. & Sales & 626 & ER & & & & \\
\hline \multicolumn{8}{|l|}{ 37. Verbenaceae } \\
\hline 136. Lantana camara $\mathrm{L}$ & Gomes & 40 & SUB & 4 & 4 & 1 & \\
\hline 137. Lippia rigida Schauer & Zickel & 9 & SUB & & 1 & & \\
\hline \multicolumn{8}{|l|}{ 38. Viscaceae } \\
\hline 138. Phoradendion piauhyanum Trel. & Pimentel & 11 & EP & & 1 & & \\
\hline \multicolumn{8}{|l|}{ 39. Zygophyllacea } \\
\hline 139. Kallstmemia tribuloide Arn.* & Villarouco & 21 & ER & & & & \\
\hline
\end{tabular}

galheirensis e Herissantia crispa (Malvaceae) e Mitracarpus hirtus e Richardia grandiflora (Rubiaceae), presentes apenas no período de disponibilidade hídrica.

A análise das 831 espécies listadas em 15 levantamentos de diferentes tipos vegetacionais encontrados no Nordeste, inclusive o da área de estudo, mostra que das 124 espécies identificadas neste trabalho, $82(66 \%)$ foram citadas em pelo menos um destes levantamentos (Tab. 1). As maiores proporções de espécies em comum, $58 \mathrm{e}$ 50 , ocorreram em levantamentos localizados em chapadas sedimentares (Rodal 1992; Andrade 1996; Oliveira et al. 1997; Figueirêdo et al. prelo), e em áreas de caatinga instaladas sobre o cristalino (Lyra 1982; Souza 1983; Fotius \& Sá 1985; Santos 1987; Rodal 1992; Alcoforado-Filho 1993; Araújo et al. 1995; Medeiros 1995; Ferraz et al. 1998), respectivamente. Os levantamentos em áreas de carrasco (Araújo et al. 1998) e cerrado (Castro 1994) apresentaram o menor número de espécies em comum com a área de estudo, 24 e nove, respectivamente.

À exceção de Andrade (1996), todos os levantamentos localizados em chapadas sedimentares foram de tipos vegetacionais arbustivos caducifólios. Merece destaque ainda, o fato dos autores desses levantamentos não classificarem a vegetação como caatinga. De modo geral, justificaram essa opção em função da flora particular, distinta da caatinga instalada no cristalino, apesar de reconhecerem que nos aspectos climático, físionômico e funcional, as áreas não diferiam de outras caatingas do cristalino.

Nessa perspectiva, procurou-se analisar a distribuição geográfica das 20 espécies citadas apenas para a área de estudo e chapadas sedimentares, a partir do rótulo das exsicatas dos herbários PEUFR e Dárdano de Andrade-Lima IPA: Conocliniopsis prasifolia e Vernonia chalybaea (Asteraceae), Arrabidea limae (Bignoniaceae), Tillandsia recurvata (Bromeliaceae), Poeppigia procera (Caesalpiniaceae), Chancesyce thymifolia e Cnidoscolus obtusifolius (Euphorbiaceae), Crotalaria bahiensis, Dalbergia cearensis e Zornia diphylla (Fabaceae), Erythroxylum revolutum (Erythroxylaceae), Hyptis martiusii (Lamiaceae), Stigmaphyllon paralias (Malpighiaceae), Piptadenia obliqua (Mimosaceae), Guapira laxa (Nyctaginaceae), Passiflora luetzelbburgii (Passifloraceae), Balfourodendron molle (Rutaceae), Cardiospermum corindum (Sapindaceae), Lippia 
Tabela 2. Famílias com maior riqueza em levantamentos florísticos e fitossociológicos realizados em diferentes tipos vegetacionais caducilólios do semi-árido nordestino e no cerrado do Estado do Plauí. TL - láxons listados e TC - táxons comuns a este trabalho

\begin{tabular}{|c|c|c|c|c|c|c|c|c|}
\hline \multirow[t]{2}{*}{ Trabalho } & \multirow{2}{*}{$\begin{array}{l}\text { Município/UF } \\
\text { Tipo de vegetação }\end{array}$} & \multicolumn{2}{|c|}{ Famílias } & \multicolumn{2}{|c|}{ Gêneros } & \multicolumn{2}{|c|}{ Espécies } & \multirow{2}{*}{$\begin{array}{l}\text { Famílias com maior } \\
\text { riqueza }\end{array}$} \\
\hline & & $\mathrm{TL}$ & $\mathrm{TC}$ & $\mathrm{TL}$ & $\mathrm{TC}$ & $\mathrm{TL}$ & TC & \\
\hline $\begin{array}{l}\text { I. Rodal } 1992 \\
\text { (PF. ME e MD) }\end{array}$ & $\begin{array}{l}\text { Floresta/Custódia. } \\
\text { PE. Caatinga }\end{array}$ & 18 & 10 & 28 & 11 & 36 & 15 & $\begin{array}{l}\text { Cactaceae, Euphorbiaceae, } \\
\text { Anacardiaceae e Caesalpiniaceae }\end{array}$ \\
\hline $\begin{array}{l}\text { 2. Ferraz et al. } 1998 \\
(500-700 \mathrm{~m})\end{array}$ & $\begin{array}{l}\text { Serra Talhada, PE. } \\
\text { Caatinga }\end{array}$ & 24 & 13 & 39 & 19 & 44 & 21 & $\begin{array}{l}\text { Euphorbiaceae, Mimosaceae, } \\
\text { Caesalpiniaceae e Anacardiaceae }\end{array}$ \\
\hline 3. Araújo et al. 1995 & $\begin{array}{l}\text { Custódia e Floresta, } \\
\text { PE. Caatinga }\end{array}$ & 20 & 10 & 38 & 12 & 47 & 17 & $\begin{array}{l}\text { Euphorbiaceae, Boraginaceae, } \\
\text { Cactaceae e Caesalpiniaceae }\end{array}$ \\
\hline $\begin{array}{l}\text { 4. Alcoforado-Filho } \\
1993\end{array}$ & $\begin{array}{l}\text { Caruaru, PE. } \\
\text { Caatinga }\end{array}$ & 33 & 11 & 67 & 16 & 75 & 18 & $\begin{array}{l}\text { Euphorbiaceae, Mimosaceae. } \\
\text { Fabaceae, Asteraceae e } \\
\text { Caesalpiniaceae }\end{array}$ \\
\hline 5. Fotius \& Sá 1985 & $\begin{array}{l}\text { Bodocó, PE. } \\
\text { Caatinga }\end{array}$ & 24 & 12 & 4.3 & 17 & 53 & 19 & $\begin{array}{l}\text { Mimosaceae, Bignoniaceae, } \\
\text { Cactaceae, Caesalpiniaceae e } \\
\text { Euphorbiaceae }\end{array}$ \\
\hline 6. Santos 1987 & $\begin{array}{l}\text { Parnamirim, PE. } \\
\text { Caatinga }\end{array}$ & 41 & 9 & 110 & 13 & 130 & 16 & $\begin{array}{l}\text { Poaceae, Euphorbiaceae, } \\
\text { Asteraceae e Caesalpiniaceae }\end{array}$ \\
\hline 7. Lyra 1982 & $\begin{array}{l}\text { Brejo da Madre de } \\
\text { Deus, PE. Caatinga }\end{array}$ & 26 & 12 & 39 & 14 & 44 & 16 & $\begin{array}{l}\text { Euphorbiaceae, Mimosaceae e } \\
\text { Cactaceae }\end{array}$ \\
\hline 8. Figueiredo et al. & $\begin{array}{l}\text { Buíque, PE. } \\
\text { Vegetação arbustiva } \\
\text { caducifólia }\end{array}$ & 35 & 21 & 73 & 31 & 94 & 38 & $\begin{array}{l}\text { Caesalpiniaceae, Euphorbiaceae, } \\
\text { Fabaceae e Mimosaceae }\end{array}$ \\
\hline 9. Medeiros 1995 & $\begin{array}{l}\text { Capistrano, CE. } \\
\text { Caatinga }\end{array}$ & 31 & 12 & 56 & 14 & 69 & 14 & $\begin{array}{l}\text { Asteraceae, Mimosaceae, } \\
\text { Euphorbiaceae e Caesalpiniaceae }\end{array}$ \\
\hline 10. Rodal 1992 (FA) & $\begin{array}{l}\text { Floresta, PE. } \\
\text { Caatinga }\end{array}$ & 10 & 5 & 19 & 7 & 22 & 8 & $\begin{array}{l}\text { Euphorbiaceae, Mimosaceae, } \\
\text { Cactaceae e Caesalpiniaceae }\end{array}$ \\
\hline 11. Souza 1983 & $\begin{array}{l}\text { Nossa Senhora da } \\
\text { Glória/Frei Paulo, } \\
\text { SE. Caatinga }\end{array}$ & 20 & 7 & 27 & 7 & 30 & 8 & $\begin{array}{l}\text { Caesalpiniaceae, Mimosaceae, } \\
\text { Rubiaceae e Cactaceae }\end{array}$ \\
\hline 12. Castro 1994 & PI. Cerrado & 52 & 7 & 148 & 8 & 249 & 8 & $\begin{array}{l}\text { Caesalpiniaceae, Mimosaceae. } \\
\text { Fabaceae, Apocynaceae e } \\
\text { Rubiaceae }\end{array}$ \\
\hline 13. Araúịo et al. 1998 & $\begin{array}{l}\text { Novo Oriente, CE. } \\
\text { Carrasco }\end{array}$ & 48 & 17 & 105 & 22 & 150 & 24 & $\begin{array}{l}\text { Caesalpiniaceae, } \\
\text { Euphorbiaceae, Mimosaceae e } \\
\text { Myrtaceae }\end{array}$ \\
\hline $\begin{array}{l}\text { 14. Oliveira et al. } \\
1997\end{array}$ & $\begin{array}{l}\text { Padre Marcos. PI. } \\
\text { Transição carrasco/ } \\
\text { caatinga de areia }\end{array}$ & 24 & 1.3 & 5.3 & 20 & 63 & 22 & $\begin{array}{l}\text { Fabaceae, Bignoniaceae, } \\
\text { Cactaceae e Caesalpiniaceae }\end{array}$ \\
\hline
\end{tabular}

rigida (Verbenaceae) e Phoradendron piauhyanum (Viscaceae). Verificou-se que todas elas têm ampla distribuição em todo o semi-árido, quer em áreas sedimentares, quer em áreas cristalinas, embora com maior freqüencia nas primeiras.

Por outro lado, foram analisadas também 30 espécies comuns à área de estudo e a levantamentos em vegetação de chapada sedimentar e caatinga instalada sobre o cristalino: Rollinia leptopetala (Annonaceae), Allamanda blanchetti e Aspidosperma pyrifolium (Apocynaceae), Cordia leucocephala (Boraginaceae), Neoglaziovia variegata (Bromeliaceae), Caesalpinia ferrea, C. microphylla, C. pyramidalis e Senna rizzini (Caesalpiniaceae), Cereus jamacaru e Opuntia inamoena (Cactaceae), Cnidoscolus quercifolius, C. urens, C. bahianus, Croton micans, Euphorbia comosa, Jatropha molissima, J. mutabilis, J. ribifolia e Manihot dichotoma (Euphorbiaceae) Bocoa mollis, Dioclea grandiflora e Zornia 
diphylla (Fabaceae), Sida galheirensis (Malvaceae), Mimosa tenuiflora, Parapiptadenia zehntneri e Piptadenia stipulacea (Mimosaceae), Richardia grandiflora (Rubiaceae), Serjania glabrata (Sapindaceae) e Lantana camara (Verbenaceae). Dessas espécies, é possível observar que Sida galheirensis, Mimosa tenuiflora e Zornia diphylla têm ampla distribuição no semiárido, especialmente em áreas antropizadas, enquanto Aspidosperma pyrifolium, Caesalpinia ferrea, C. pyramidalis, Cereus jamacaru, Parapiptadenia zehntneri são mais freqüentes no cristalino e Caesalpinia microphylla. Senna rizzini, Cnidoscolus bahianus, Jatropha mutabilis e Bocoa mollis, mais comuns em áreas sedimentares.

Os dados de distribuição de boa parte das espécies registradas na área de estudo indicam tratar-se de de conjunto florístico comum às áreas cristalinas e sedimentares, embora com maior frequiência nas últimas.

Apesar de Rodal (1992) observar que a similaridade florística entre levantamentos de caatinga está ligada primeiramente à proximidade geográfica e, em seguida, ao grau de deficiência hídrica, isso não foi observado neste levantamento. As similaridades foram muito mais relacionadas às variações pedológicas e topográficas, do mesmo modo que o observado por Pagano et al. (1995) para as florestas mesófilas do interior do Estado de São Paulo. Vale salientar que Rodal (1992) analisou unicamente levantamentos em áreas do cristalino.

Pode-se concluir que, embora a flora da área de estudo represente conjunto florístico mais especializado a ambientes com solos arenosos e profundos, essas espécies também ocorrem, embora com menor frequiência, em áreas de caatinga instaladas sobre o cristalino. Assim, a flora foi considerada como pertencente à vegetação de caatinga.

\section{Agradecimentos}

À equipe do projeto "Espécies lenhosas da caatinga de Pernambuco" da Universidade Federal Rural de Pernambuco, em especial à Professora Luzimar Bastos Paes e Mello, pelo processamento da coleção no herbário PEUFR.

\section{Referências bibliográficas}

Albuquerque, S. G.; Soares, J. G. G.; Araújo Filho, J. A. 1982. Densidade de espécies arbustivas em vegetação de caatinga. (Pesquisa em andamento, 16). EMBRAPACPATSA. Petrolina.

Alcoforado-Filho, F. G. 1993. Composição florística e fitossociologia de uma área de caatinga arbórea no município de Caruaru-PE. Dissertação de Mestrado. UFRPE. Recife.

Andrade. G. O. de. 1977. Alguns aspectos do quadro natural do Nordeste. SUDENE. Coordenação de planejamento regional - Divisão de Política Espacial, Recife.

Andrade, K. V. S. A. 1996. Florística e fitossociologia de um refúgio vegetacional no município de Buíque Pernambuco. Monografia de Graduação. UFRPE, Recife.

Andrade-Lima, D. de. 1960. Estudos fitogeográficos de Pernambuco. $2^{\text {a }}$ ed. revista. Arquivos do Instituto de Pesquisas Agronômicas 5: 305-341.

Andrade-Lima. D. de. 1964. Notas para a fitogeografia de Grossos. Mossoró e Areia Branca. Anais da Associação de Geógrafos Brasileiros 12: 29-48

Andrade-Lima. D. de. 1966. Vegetação. In: IBGE. Atlas Nacional do Brasil. Conselho Nacional de Geografia. Recife.

Andrade-Lima. D. de. 1978. Vegetação. Pp. 123-135. In: Lins. R.C. Bacia do Parnaíba: aspectos fisiográficos. (Série Estudos e Pesquisas, 9). Instituto Joaquim Nabuco de Pesquisas Sociais, Recife.

Andrade-Lima. D. de. 1981. The caatinga dominium. Revista Brasileira Botânica 4: 149-53.

Araújo. E. L.: Sampaio, E. V. S. B. \& Rodal, M. J. N. 1995. Composição florística e fitossociológica de três áreas de calatinga. Revista Brasileira de Biologia 55(4): 595-607.

Araújo. F. S.: Sampaio, E. V. S. B.; Rodal, M. J. N. \& Figueirêdo, M. A. 1998. Organização comunitária do componente lenhoso de três áreas de carrasco em Nova Oriente - CE. Revista Brasileira de Biologia 58(1): 85-95.

Araújo. F. S.: Sampaio, E. V. S. B.; Figueiredo, M. A.; Rodal. M. J. N. \& Fernandes, A. G. 1998. Composição florística da vegetação de carrasco, Novo Oriente - CE. Revista Brasileira de Botânica 21(2):15-26.

Barroso, G. M.: Guimarães, E. F.; Ichaso, C. L. F.; Costa, C. G. E \& Peixoto, A. L. 1978. Sistemática de Angiospermas do Brasil: I. LCT/EDUSP, São Paulo.

Barroso, G. M.; Peixoto, A. L.; Costa, C. G.; Ichaso, C. L. F. \& Guimarães, E. F. 1984. Sistemática de Angiospeermas do Brasil: II. UFV, Imprensa Universitária, Viçosa.

Barroso. G. M.: Peixoto, A. L.; Costa, C. G.; Ichaso, C. L. F. \& Guimarães. E. F. 1986. Sistemática de Angiospermas do Brasil: III. UFV, Imprensa Universitária, Viçosa.

Brasil - Ministério de Minas e Energia. 1981. Vegetação. Pp.405-456. In: Folha SD.2Y. Salvador: Geologia, geomorfologia, pedologia, vegetação, uso e potencial da terra. (Levantamento Recursos Naturais 2). Rio de Janeiro.

Bridson. D. \& Forman, L. 1992. The herbarium handbook. Royal Botanic Gardens, Kew. 
Brito. I. M. 1979. Bacias sedimentares e formações póspaleozóicas do Brasil. Interciência. Rio de Janeiro.

Brummitt. R. K. 1992. Vascular plant families and genera. Royal Botanic Gardens, Kew.

Brummitt. R. F. \& Powell, C. E. 1992. Authors of plant names. Royal Botanic Gardens, Kew.

Castro, A. A. J. F. 1994. Comparação florístico-geográfica (Brasil) e fitossociológica (Piauí - São Paulo). Tese de Doutorado. UNICAMP, Campinas.

Cronquist. A. 1988. The evolution and classification of flowering plants. New York Botanical Garden, New York.

Dantas. J. R. A. 1980. Mapa geológico do Estado de Pernambuco. DNPM, Recife.

Drumond, M. A.: Lima, P. C. F; Souza, S. M. de \& Lima, J. L. S. de. 1979. Sociabilidade de algumas espécies que ocorrem na caatinga. Pp. 32-33. In: Anais do Congresso Nacional de Botânica. Sociedade Botânica do Brasil.

Egler. W. A. 1951. Contribuição ao estudo da caatinga pernambucana. Revista Brasileira de Geografia 13(4): $65-77$.

Emperaire. L. 1991. Vegetação e flora. Pp. 61-206. In: IBAMA. Plano de manejo: Parque Nacional da Serra da Capivara. Brasília.

Fernandes. A. G.1996. Fitogeografia do semi-árido. Pp. 215219. Anais da $4^{\text {a }}$ Reunião Especial da Sociedade para o Progresso da Ciência. SBPC. Feira de Santana.

Fernandes, A. G. \& Bezerra, P. 1990. Estudo fitogeográfico do Brasil. Editora Styllus Comunicações, Fortaleza.

Ferraz. E. M. N.: Rodal, M. J. N.; Sampaio, E. V. S. B. \& Pereira, R. de C. A. 1998. Composição florística em trechos de vegetação de caatinga e brejo de altitude na região do Vale do Pajeú, Pernambuco. Revista Brasileira de Botânica 21(1): 7-15.

Ferreira, R. L. C. 1988. Análise estrutural da vegetação da estação florestal de experimentação de Açu-RN, como subsídio básico para o manejo florestal. Dissertação de Mestrado. UFV, Viçosa.

Ferreira. P. A. \& Cadier, E. 1978. Bacia representativa de Ihipeba: campanha 1976/1977. SUDENE-DRN, SUDENE. Recife. Hidrologia 6: $81 \mathrm{p}$.

Figueirêdo, L. S: Rodal, M. J. N. \& Melo. A. L. prelo. Florística e fitossociologia de uma área de vegetação arbustiva caducifólia no município de Buíque - Pernambuco. Naturalia.

Figueiredo. M. A. 1987. A microrregião salineira norteriograndense no domínio das caatingas. Mossoró, Escola Superior de Agricultura de Mossoró. Coleção Mossoroense. 353; 44p.

Fonseca. M. R. da. 1991. Análise da vegetação arbustivoarbórea da caatinga hiperxerófila do nordeste de Sergipe. Tese de Doutorado. UNICAMP, Campinas.

Font-Quer. M. P. 1977. Diccionario de botánica. Lábor, Barcelona.

Fotius. G. A. \& Sá. I. B. 1985. Esboço da vegetação da bacia hidrográfica da Sipaúba, Bodocó, PE. EMBRAPACPATSA. Petrolina.

Gentry. A. H. 1995. Diversity and floristic composition of neotropical dry forests. Pp.146-194. In: S. H. Bullock:
H. A. Mooney \& E. Medina, E. Seasonal Dry Tropical Forests, Cambridge University Press, Cambridge.

Gomes. M. A. F. 1979. Padrões de caatinga nos Cariris Velhos, Paraíba. Dissertação de Mestrado. UFRPE. Recife.

Jacomine, P. T.; Cavalcanti, A. C.; Burgos, N.: Pessoa, S. C. P. \& Silveira, C. O. 1973. Levantamento exploratórioreconhecimento de solos do Estado de Pernambuco. Divisão de Pesquisa Pedológica. V. I (Boletim Técnico 26 Pedologia 14). Recife.

Lyra, A. L. R. T. 1982. Efeito do relevo na vegetação de duas áreas do município do Brejo da Madre de Deus (PE). Dissertação de Mestrado. UFRPE, Recife.

Martius. C. F. P. von \& Eichler, A. G. 1840/1906. Flora Brasiliensis: enumeratio plantarum in Brasilia hactenus detectarum. Monachii. Lipsiae. Reimpressão 1965. Verlag von J. Cramer. 15 v.

Medeiros, J. B. L. de P. 1995. Florística e fitossociologia de uma área de caatinga localizada na fazenda Aracangá, Município de Capristano - CE. Monografia de Graduação. UFCE. Fortaleza.

Mello-Neto. A. V.: Lins, R. C. \& Coutinho, S. F. 1992. Áreas de exceçâo úmidas e subúmidas do semi-árido do nordeste do Brasil: estudo especial. In: Impactos de variações climáticas e desenvolvimento sustentável em regiōes semi-áridas. Fundação Joaquim Nabuco, Recife.

Moreira. A. A. N. 1977. Relevo. Pp.1-45. In: IBGE Geografia do Brasil. Região Nordeste. Rio de Janeiro.

Nimer. E. 1979. Climatologia do Brasil. Fundação IBGESUPREN. (Recursos Naturais e Meio Ambiente 4). Rio de Jaineiro.

Oliveira. J. G. B. de; Quesado, H. L. S.; Nunes, E. P.: Figueiredo. M. A. \& Bezerra, C. L. F. 1988, Vegetação da estação ecológica de Aiuaba, Ceará. Mossoró, Escola Superior de Agricultura de Mossoró. Coleção Mossoroense. 357 B: 27p.

Oliveira. M. E. A.: Sampaio, E. V. S. B.; Castro, A. A. J, de F. \& Rodal. M. J. N. 1997. Flora e fitossociologia de uma área de transição carrasco-caatinga de areia em Padre Marcos. Piauí. Naturalia 22: 131-150.

Pagano, S. N.; Leitão-Filho, H. F. \& Cavassan, O. 1995. Variaçĩo temporal da composição florística e estrutura fitossociológica de uma floresta mesófila semi-decídua, Rio Claro. Estado de São Paulo, Revista Brasileira de Biologia 55(2): 24I-258.

Reis, A. C. de S. 1976. Clima da caatinga. Anais da Academia Brasileira de Ciências 48(2): 325-335.

Rizzini. C. T. 1979. Tratado de fitogeografia do Brasil. HUCITEC/EDUSP. V.2, São Paulo.

Rodal. M. J. N.. 1983. Fitoecologia de uma área do médio vale do Moxotó, Pernambuco. Dissertação de Mestrado. UFRPE. Recife.

Rodal. M. J. N. 1992. Fitossociologia da vegetação arbustivo-arbórea em quatro áreas de caatinga $\mathrm{em}$ Pernambuco. Tese de Doutorado. UNICAMP, Campinas.

Rodal. M. J. N.: Andrade, K. V. de S. A.; Sales, M. F. de \& Gomes. A. P. S. 1998. Fitossociologia do componente lenhoso de um refúgio vegetacional no município de Buíque. Pernambuco. Revista Brasileira de Biologia 58(3): $517-526$. 
Santos. M. F. A. V. 1987. Características de solo e vegetação em sete áreas de Parnamirim, Pernambuco. Dissertação de Mestrado. UFRPE. Recife.

Souza, G. V. 1983. Estrutura da vegetação da caatinga hipoxerófila do Estado de Sergipe. Dissertação de Mestrado. UFRPE, Recife.

Souza. M. J. N. de; Martins, M. L. R.; Soares, Z. M. L.; FreitasFilho, M. R. de; Almeida, M. A. G. de: Pinheiro, F. S. de A.: Sampaio, M. A. B.; Carvalho, G. M. B. S.; Soares, A. M. L.: Gomes, E. C. B. \& Silva, R. A. 1994. Redimensionamento da região semi-árida do Nordeste do Brasil. In: Conferência Nacional e Seminário Latino-Americano de Desertificação. Fundação Esquel do Brasil, Fortaleza.

Stafleu. F. A. \& Cowan, R. S. 1976/1988. Taxonomic Literature. Scheltema \& Holkema. 7 v., Utrecht.

Streillein. K.E. 1982. Ecology of small mammals in the semiarid Brazilian caatinga. I- Climate and faunal composition. Annals of Carnigie Museum of Natural History 51: 79-107.

SUDENE. Supertintendência para o Desenvolvimento do Nordeste. 1979. Projeto para o desenvolvimento integrado da Bacia hidrogeológica do Jatobá: levantamento dos recursos da vegetação. Recife. 46p.

Tavares, S.: Paiva, F. A. V.; Tavares, E. J. de S.; Carvalho, G. H. \& Lima. J. L. S. de. 1969a. Inventário florestal do Ceará. 1. Estudo preliminar das matas remanescentes do município de Quixadá. Boletim de Recursos Naturais 7(1/4): 93-111.

Tavares, S.: Paiva, F. A. V.; Tavares, E. J. de S.; Lima, J. L. S. de. \& Carvalho, G. H. 1969b. Inventário florestal de
Pernambuco. Estudo preliminar das matas remanescentes do município de São José do Belmonte. Boletim Recursos Naturais 7(1/4): 113-139.

Tavares. S.: Paiva. F. A. V.; Tavares, E. J, de S.; Carvalho, G. H. \& Limal. J. L. S. de. 1970. Inventário florestal de Pernambuco. Estudo preliminar das matas remanescentes do município de Ouricuri, Bodocó. Santa Maria da Boa Vista e Petrolina. Boletim Recursos Naturais 8(1/2): 149194.

Tavares, S.; Paiva, F. A. V.; Tavares, E. J. de S. \& Lima, J. L. S. de. 1974a. Inventário florestal do Ceará 11. Estudo preliminar das matas remanescentes do município de Tauá. Boletim de Recursos Naturais 12(2): 5-19.

Tavares. S.: Paiva. F. A. V.; Tavares, E. J. de S. \& Lima. J. L. S. de. 1974b. Inventário florestal do Ceará IIl . Estudo preliminar das matas remanescentes do município de Barbalha. Boletim de Recursos Naturais 12(2): 2046

Tavares, S.: Paiva, F. A. V.; Tavares, E. J. de S. \& Carvalho, G. H. 1975. Inventário florestal da Paraíba e no Rio Grande do Norte l. Estudo preliminar das matas remanescentes do vale do Piranhas. Recife. SUDENE. 31 p. (Recursos Naturais 3).

Vasconcelos-Sobrinho, J. 1941. As regiões naturais de Pernambuco. Arquivos do Instituto de Pesquisas Agrônomicas 3: 25-32.

Walter . H. \& Lieth, M. 1964. Die vegetacion der erde. Band. Die tropischen und subtropischen zonen. Gustav Fischer Verlag, Sttutgart. 